\title{
DUAL VECTOR FIELDS II: CALCULATING THE JACOBIAN
}

\author{
PHILIP FEINSILVER \\ Department of Mathematics \\ Southern Illinois University \\ Carbondale, IL 62901, U.S.A. \\ E-mail:pfeinsil@math.siu.edu \\ RENÉ SCHOTT \\ IECN and LORIA \\ Université Henri Poincaré-Nancy 1, BP 239 \\ 54506 Vandoeuvre-lès-Nancy, France \\ E-mail:schott@loria.fr
}

\begin{abstract}
Given a Lie algebra with a chosen basis, the change of coordinates relating coordinates of the first and second kinds near the identity of the corresponding local group yields some remarkable vector fields and dual vector fields. One family of vector fields is dual to a representation of the Lie algebra acting on a Fock-type space. To this representation an abelian family of dual vector fields is associated. The exponential of these commuting operators acting on an appropriate vacuum yields the same result as does the local group element generated by the nonabelian Lie algebra. Another family of dual vector fields gives a representation of the Lie algebra, yet acting on an appropriate vacuum, yields the same result as an abelian Lie algebra. An essential component of these constructions is the Jacobian of the change of coordinates. Here we present a formula for this Jacobian using the pi-matrices that play a fundamental rôle in our approach to representations of Lie algebras.
\end{abstract}

1. Introduction. We continue the study of dual vector fields [2]. The main features of our approach to the representations of Lie algebras may be summarized as follows. For a given Lie algebra with a chosen basis, we find two families of vector fields corresponding to left and right multiplication respectively of the Lie algebra on the local Lie group near the identity. The left action is dual to a representation of the Lie algebra and leads to the double dual representation. The coefficients of these vector fields form matrix-valued

2000 Mathematics Subject Classification: Primary 22E05.

The paper is in final form and no version of it will be published elsewhere. 
functions on the group, these are the fundamental pi-matrices. In [2], a basic rôle is played by the Jacobian of the change of coordinates on the local group from first to second kind. In this article, we present a method for finding the Jacobian directly in terms of the pi-matrices evaluated along a path in the local group.

In [2], we outlined a nine-step program for finding, for any given Lie algebra in a chosen basis, an associated family of polynomials, and corresponding commuting operatorsdual vector fields - which yield the coordinates of the second kind when acting on an appropriate vacuum, and, as well, a realization of the Lie algebra as dual vector fields that when acting on an appropriate vacuum state yield a pure exponential, the same as an abelian Lie algebra.

The basic steps are these (to be explained in detail in the next section):

1. Given a Lie algebra with a chosen basis.

2. Find the pi-matrices. From $\pi^{\ddagger}$, form the double dual.

3. Solve the equations $\dot{A}=\alpha \pi^{\ddagger}$ and find the mapping from coordinates of the first kind to coordinates of the second kind.

4. Identify canonical variables for associating an abelian family of dual vector fields.

5. Calculate the Jacobians $\partial A / \partial \alpha$ and $\partial \alpha / \partial A$. $\partial A / \partial \alpha$ is used to find the raising operators $\hat{Y}$, whereas $\partial \alpha / \partial A$ yields recurrence relations for the canonical polynomials.

6. Specifically, $\hat{Y}=x A^{\prime}(\alpha(A)), x=\hat{Y} A^{\prime}(\alpha)^{-1}$.

7. Canonical polynomials are $\eta_{n}(x)=\hat{Y}^{n} 1$. The $\hat{Y}$ variables act as raising operators via $\hat{Y}=x A^{\prime}(\alpha(\partial))$. Corresponding lowering operators are $\mathcal{V}_{i}$. Recursion formulas are given via $x=\hat{Y} A^{\prime}(\mathcal{V})^{-1}$.

8. Performing a local analytic change of variables yields dual vector fields $\hat{\xi}_{i}$.

9. Specifically, using $A$ as a change of variables yields a realization of the Lie algebra as dual vector fields

$$
\hat{\xi}=x_{\nu} A^{\prime}(\partial)_{\nu \lambda}^{-1} \pi_{i \lambda}^{\ddagger}(A(\partial))
$$

satisfying

$$
e^{\alpha_{\mu} \hat{\xi}_{\mu}} 1=e^{\alpha_{\mu} x_{\mu}}
$$

In the following section, we will discuss the above steps so that this article may be read independently, referring the reader to [2] for proofs. Then we present our main theorem, giving a (new) formula for the Jacobian of the coordinates. We will illustrate with two fundamental Lie algebras whose detailed study illuminates the theory: the twodimensional affine Lie algebra and the finite difference algebra. (Note: details for the Heisenberg algebra have been given in [2].)

Notation. We use a summation convention somewhat more general than that of the Einstein convention, namely, Greek indices are always summed, regardless of position. Latin indices are summed only if the summation is indicated explicitly.

2. Lie algebras, vector fields, and dual vector fields. In this section, we present the background and basic constructions of interest.

First, the definition of a dual vector field. 
REMARK. Note that throughout we are working with analytic vector fields, i.e., the coefficients are analytic in some neighborhood of 0 in $\mathbf{C}^{N}$.

Definition. A dual vector field, dvf for short, is an operator dual to a vector field. I.e., an operator of the form

$$
\hat{X}=x_{\mu} F_{\mu}(\partial)
$$

where $F: \mathbf{C}^{N} \rightarrow \mathbf{C}^{N}$ is holomorphic in a neighborhood of the origin in $\mathbf{C}^{N}$.

2.1. Lie algebras and dual representations. Start with a finite-dimensional Lie algebra with basis $\left\{\xi_{1}, \ldots, \xi_{N}\right\}$. Define (local) group elements

$$
g(A, \xi)=e^{A_{1} \xi_{1}} \cdots e^{A_{N} \xi_{N}} .
$$

The fundamental theorems of Lie imply that there is a group law

$$
g(A, \xi) g(B, \xi)=g(A \odot B, \xi)
$$

where $A \odot B$ is analytic in the variables $A=\left(A_{1}, \ldots, A_{N}\right), B=\left(B_{1}, \ldots, B_{N}\right)$. Note that $g(0, \xi)$ is the identity.

Further, Lie's fundamental theorems (Frobenius integrability theory) imply that there is a realization of the $\xi_{i}$ acting on the group elements $g(A, \xi)$ by multiplication on the left as vector fields $\xi_{i}^{\ddagger}$. Namely,

$$
\xi_{i} g(A, \xi)=\xi_{i}^{\ddagger} g(A, \xi)=\left(\pi_{i \mu}^{\ddagger}(A) \partial_{\mu}\right) g(A, \xi) .
$$

[Throughout this section, $\partial$ will denote partial differentiation with respect to the $A$ variables: $\partial_{i}=\partial / \partial A_{i}$.]

There is a similar action on the right:

$$
g(A, \xi) \xi_{i}=\xi_{i}^{*} g(A, \xi)=\left(\pi_{i \mu}^{*}(A) \partial_{\mu}\right) g(A, \xi) .
$$

These vector fields thus define the pi-matrices: $\pi^{\ddagger}$ and $\pi^{*}$ as the corresponding coefficients.

One way to see what is going on is to expand $g$ in powers of the $A$ variables, showing $g$ as the generating function of the Poincaré-Birkhoff-Witt (PBW) basis of the universal enveloping algebra:

$$
g(A, \xi)=\sum_{n \geq 0} \frac{A^{n}}{n !} \xi_{1}^{n_{1}} \cdots \xi_{N}^{n_{N}}
$$

so that left-multiplication by $\xi_{i}$ is dual to the action of the vector field $\xi_{i}^{\ddagger}$ in the sense that the action of $\xi$ on the PBW basis is transferred via the generating function $g$ to a differential operator acting on functions of the $A$-variables. And similarly for rightmultiplication.

Another way to interpret this action leads to the double dual. Introduce canonical bosons acting on the PBW basis thus:

$$
\begin{aligned}
\mathcal{R}_{i} \xi_{1}^{n_{1}} \cdots \xi_{i}^{n_{i}} \cdots \xi_{N}^{n_{N}} & =\xi_{1}^{n_{1}} \cdots \xi_{i}^{n_{i}+1} \cdots \xi_{N}^{n_{N}}, \\
\mathcal{V}_{i} \xi_{1}^{n_{1}} \cdots \xi_{i}^{n_{i}} \cdots \xi_{N}^{n_{N}} & =n_{i} \xi_{1}^{n_{1}} \cdots \xi_{i}^{n_{i}-1} \cdots \xi_{N}^{n_{N}} .
\end{aligned}
$$

These are raising and lowering operators for the PBW-basis. Then the action of a vector field $\xi_{i}^{\ddagger}=\pi_{i \mu}^{\ddagger}(A) \partial_{\mu}$ dualizes to the dual vector field $\hat{\xi}_{i}=\mathcal{R}_{\mu} \pi_{i \mu}^{\ddagger}(\mathcal{V})$. This is the double dual representation. It is a boson realization of the action of left multiplication of the 
basis elements $\xi_{i}$ on the PBW basis. The important feature is that since the left action is an anti-homomorphism, the double dual gives a homomorphism of the Lie algebra.

Remark. For more details see [2, pp. 28-30 (Chapter 2)]. The double dual is found in [2, pp. 35-36/40].

Now let $X=\alpha_{\mu} \xi_{\mu}$ be a typical element of the Lie algebra. The $\alpha$-variables are coordinates of the first kind. Define the coordinate mapping $\alpha \rightarrow A$, the coordinates of the second kind, by

$$
\exp (X)=\exp \left(\alpha_{\mu} \xi_{\mu}\right)=e^{A_{1}(\alpha) \xi_{1}} \cdots e^{A_{N}(\alpha) \xi_{N}}=g(A(\alpha), \xi) .
$$

If we consider the one-parameter subgroup generated by $X$, we have

$$
\exp (t X)=g(A(t), \xi)
$$

where $A(t)$ is implicitly a function of $\alpha$. It is important to note that the $t$-dependence comes from scaling $\alpha \rightarrow t \alpha$. In particular, if the mapping $A(\alpha)$ is known, then $A(t)=$ $A(t \alpha)$ and conversely, given $A(t)$ we recover $A(\alpha)=\left.A(t)\right|_{t=1}$.

Differentiating with respect to $t$ provides two choices: we can multiply by $X$ on the left or on the right. In each case, the action of the $\xi_{i}$ can be converted to the corresponding vector fields. Notice that, on $g(A, \xi)$, the operator $\partial_{i}$ brings down the corresponding $\xi_{i}$ in place, i.e., to bring it to the front or back would require using the adjoint action of the (partial products) group elements. The left/right vector fields do this automatically. E.g., bringing down $X$ on the left yields

$$
X e^{t X}=\alpha_{\mu} \xi_{\mu}^{\ddagger} g(A, \xi)=\dot{A}_{\mu} \partial_{\mu} g(A, \xi) .
$$

We read off the characteristic equations for the flow:

$$
\dot{A}_{i}=\alpha_{\lambda} \pi_{\lambda i}^{\ddagger}(A) \text {. }
$$

However, we can just as well bring down $X$ on the right, yielding

$$
\dot{A}_{i}=\alpha_{\lambda} \pi_{\lambda i}^{*}(A)
$$

Solving these equations with zero initial conditions, $A_{i}(0)=0,1 \leq i \leq N$, corresponding to the identity of the group, will yield $A(t)$, functions of $t$ and $\alpha$. Setting $t=1$ then gives the coordinate map $\alpha \rightarrow A$.

To conclude this part, recall the adjoint representation of the Lie algebra, acting on itself by Lie bracket (commutation): $(\operatorname{ad} \xi)(\zeta)=[\xi, \zeta]$. With the basis $\left\{\xi_{i}\right\}$, we denote the matrices of the adjoint representation of the basis elements by $\check{\xi}_{i}$. The corresponding group element, exponential of the adjoint representation, is

$$
\check{\pi}(A)=\exp \left(A_{1} \check{\xi}_{1}\right) \cdots \exp \left(A_{N} \check{\xi}_{N}\right) .
$$

Notation. We use ^ 's to denote the transposed pi-matrices. Namely (dropping the dagger for convenience), $\hat{\pi}$ is the transpose of $\pi^{\ddagger}$, and $\hat{\pi}^{*}$ is the transpose of $\pi^{*}$.

We have the relation ([2, pp. 36-37]):

$$
\check{\pi}=\hat{\pi}^{-1} \hat{\pi}^{*} \text {. }
$$

We have the first three basic steps in hand. The next part will discuss the remaining aspects. 
2.2. Associated abelian variables. Given

$$
V: \mathbf{C}^{N} \rightarrow \mathbf{C}^{N}, \quad V(z)=\left(V_{1}\left(z_{1}, \ldots, z_{N}\right), \ldots, V_{N}\left(z_{1}, \ldots, z_{N}\right)\right)
$$

holomorphic in a neighborhood of the origin, satisfying $V(0)=0$, we construct a corresponding abelian family of dual vector fields. We interpret the variables $z_{i}$, as lowering operators (by partial differentiation) acting on functions of variables $x_{j}$, raising operators. The sets of variables $\left\{z_{j}\right\},\left\{x_{i}\right\}$ are canonical conjugates, i.e., they satisfy the canonical commutation relations, $\mathrm{CCR},\left[z_{i}, x_{j}\right]=\delta_{i j},\left[z_{i}, z_{j}\right]=\left[x_{i}, x_{j}\right]=0$. We think of $z_{i}$ as the partial derivative operator $\partial / \partial x_{i}$ acting on germs of holomorphic functions of $\left(x_{1}, \ldots, x_{N}\right)$. Another terminology is that they are standard boson operators: creation/raising (modelled as multiplication by $x_{i}$ ) and annihilation/lowering (modelled as partial differentiation $\left.z_{i}\right)$ operators. In this context, a function of $x=\left(x_{1}, \ldots, x_{N}\right), f(x)$, is identified with $f(x) 1$, the operator of multiplication by $f(x)$ acting on the vacuum state 1 , with $z_{i} 1=0$, for all $1 \leq i \leq N$.

Denoting the Jacobian $V^{\prime}(z)$, let $W(z)=\left(V^{\prime}(z)\right)^{-1}$, be the inverse (matrix inverse) Jacobian. Then the CCR extend to $\left[V(z), x_{i}\right]=\partial V / \partial z_{i}$ and thus, setting the operators

$$
\hat{Y}_{i}=x_{\mu} W_{\mu i}(z)
$$

yields the system of raising and lowering operators $\left\{\hat{Y}_{j}\right\},\left\{V_{i}\right\}, 1 \leq i, j \leq N$, with $\left[V_{i}, \hat{Y}_{j}\right]=\delta_{i j}$. The essential feature, which one checks, is that, $\left[\hat{Y}_{i}, \hat{Y}_{j}\right]=\left[V_{i}, V_{j}\right]=0$. Notice that exchanging $z$ with $x$ is a Fourier transformation and turns the variables $\hat{Y}_{i}$ into the vector fields $Y_{i}=W(x)_{\mu i} \partial / \partial x_{\mu}$. Thus, the $\hat{Y}_{i}$ are dual vector fields. Here, the ^ notation for dual vector fields refers to the formal Fourier transformation.

Notation. We complement the standard notations used along with $V$ and $W$, letting $U$ denote the inverse function to $V$. I.e., $U \circ V=V \circ U=$ id. Explicitly: $U(V(z))=z$.

Observe that since $W=V^{\prime-1}$, we have $W(z)=U^{\prime}(V(z))$. In other words, converting from $z$ to $V$ acting on functions of the canonical variables $\hat{Y}_{i}$, we have $x=\hat{Y} U^{\prime}(V)^{-1}$.

The formula we want ([1, p. 185, eq. (1)]) is

$$
\exp \left(\alpha_{\mu} \hat{Y}_{\mu}\right) 1=\exp \left(x_{\mu} U_{\mu}(\alpha)\right)=\sum_{n \geq 0} \frac{\alpha^{n}}{n !} \eta_{n}(x),
$$

the $n$ denoting a multi-index $\left(n_{1}, \ldots, n_{N}\right)$. This expansion defines the canonical polynomials: $\eta_{n}(x)=\hat{Y}^{n} 1$.

In our context, $A$ corresponds to $U$ and $\alpha$ to $V$. That is, $\alpha(A)$, the map $A \rightarrow \alpha$, as $V$ with $A$ instead of $z$. Form the canonical variables $\hat{Y}_{i}=x_{\mu} W_{\mu i}(\partial / \partial x)$, where $W=\alpha^{\prime}(A)^{-1}$ is the inverse Jacobian matrix of the map. Alternatively, we can express this using the Jacobian $A^{\prime}(\alpha)$, re-expressed in terms of the $A$-variables, then replacing every $A_{i}$ by the partial differentiation operator $\partial / \partial x_{i}$.

Now, write the double dual $\hat{\xi}_{i}=\mathcal{R}_{\mu} \pi_{i \mu}^{\ddagger}(\mathcal{V})$ in variables $\mathcal{R} \leftrightarrow x$ and $\mathcal{V} \leftrightarrow \partial / \partial x$, $\hat{\xi}_{i}=x_{\mu} \pi_{i \mu}^{\ddagger}(\partial / \partial x)$. Then we have

$$
\exp \left(\alpha_{\mu} \hat{\xi}_{\mu}\right) 1=\exp \left(x_{\mu} A_{\mu}(\alpha)\right)=\exp \left(\alpha_{\mu} \hat{Y}_{\mu}\right) 1
$$

in other words, the commuting dual vector fields $\left\{\hat{Y}_{i}\right\}$ yield the same result on the vacuum state as does the group element in the double dual representation. In both cases, we have 
the coordinate map $\alpha \rightarrow A$, which contains the basic information on the structure of the Lie algebra (e.g., we can recover the adjoint representation).

Introduce canonical conjugates to $\left\{\hat{Y}_{i}\right\}:\left\{\mathcal{V}_{j}\right\}$. Then we have, with $x$ as a row vector,

$$
\hat{Y}=x A^{\prime}(\alpha(\partial)), \quad x=\hat{Y} A^{\prime}(\mathcal{V})^{-1},
$$

partials here denoting differentiation with respect to $x$. The variables $\hat{Y}_{i}$ are raising operators, generating canonical polynomials $\eta_{n}(x)=\hat{Y}^{n} 1$. Thus the Lie algebra has an associated family of polynomials. The $\mathcal{V}_{i}$ act as formal differentiation operators: $\mathcal{V}_{i} \eta_{n}=n_{i} \eta_{n-e_{i}}$. Thus, the action of the variables $x_{i}$ yields recurrence formulas for the polynomials $\eta_{n}$, by expansion in powers of the $\mathcal{V}_{i}$.

One can do a change of variables in the left and right representations of the Lie algebras as well. For example, we can form the vector fields

$$
\xi_{i}^{\ddagger}=\pi_{i \lambda}^{\ddagger}(V(x)) W_{\nu \lambda}(x) \partial / \partial x_{\nu}
$$

corresponding to dual vector fields

$$
\hat{\xi}_{i}=x_{\nu} W_{\nu \lambda}(\partial) \pi_{i \lambda}^{\ddagger}(V(\partial)) .
$$

And with $\hat{X}=\alpha_{\mu} \hat{\xi}_{\mu}$

$$
e^{\hat{X}} 1=e^{x U(A(\alpha))} .
$$

Now choose $U$ and $A$ to be inverse maps. Then

$$
\hat{\xi}_{i}=x_{\nu} A^{\prime}(\partial)_{\nu \lambda}^{-1} \pi_{i \lambda}^{\ddagger}(A(\partial)) .
$$

Then we have the nonabelian Lie algebra yielding the same result on the vacuum state, 1 , as the abelian one, namely

$$
\exp \left(\alpha_{\mu} \hat{\xi}_{\mu}\right) 1=\exp \left(\alpha_{\mu} x_{\mu}\right)
$$

That completes our summary of the basic features involved.

3. Jacobian of the coordinate map. To get the canonical variables requires the Jacobian of the map $\alpha \rightarrow A$. Since one has the differential equations for $A$, namely the characteristic equations $\dot{A}=\alpha \pi(A)$, one would think it possible to find $A^{\prime}(\alpha)=\partial A / \partial \alpha$ directly in terms of the $\pi$-matrices. This turns out to be the case and is the subject of our main theorem. Namely

Theorem 1. Let $J=\partial A / \partial \alpha$ denote the Jacobian of the coordinate map $\alpha \rightarrow$ A. Then

$$
J(\alpha)=\hat{\pi}(A(\alpha)) \int_{0}^{1} \check{\pi}(A(s)) d s .
$$

Alternatively, we have

$$
J(\alpha)=\hat{\pi}^{*}(A(\alpha)) \int_{0}^{1} \check{\pi}^{-1}(A(s)) d s .
$$

We will give the proof in several steps.

Note that in our application to dual vector fields, we really want $J$ as a function of $A$. The factor outside the integral is naturally given in terms of the $A_{i}$, but the integral is evaluated by expressing $A(s)$ in terms of the $\alpha_{i}$ scaled by $s$. On the other hand, $J^{-1}$ 
immediately gives us what we need for the corresponding $x$-variables (see examples in the next section).

Definition. 1. Since $\dot{A}=\alpha \pi^{*}(A)=\alpha \pi^{\ddagger}(A)$, we will use $\tilde{\pi}$ when either the left or right pi-matrix will serve.

2. In the rest of this section, $\partial$ and $\partial_{i}$ are with respect to the $A$-variables.

3. The Jacobian matrix $\partial \dot{A} / \partial A$ arises as follows. Starting with $\dot{A}_{i}=\alpha_{\mu} \tilde{\pi}_{\mu i}$, apply $\partial_{j}$ to both sides. Then

$$
\left(\frac{\partial \dot{A}}{\partial A}\right)_{i j}=\alpha_{\mu} \frac{\partial \tilde{\pi}_{\mu i}}{\partial A_{j}}
$$

To specify left or right, we will use

$$
\left(\frac{\partial \dot{A}^{\ddagger}}{\partial A}\right)_{i j}=\alpha_{\mu} \frac{\partial \pi_{\mu i}^{\ddagger}}{\partial A_{j}} \quad \text { and } \quad\left(\frac{\partial \dot{A}^{*}}{\partial A}\right)_{i j}=\alpha_{\mu} \frac{\partial \pi_{\mu i}^{*}}{\partial A_{j}}
$$

Define $J(t)=\partial A(t \alpha) / \partial \alpha$. Then we obtain $J$ as $\left.J(t)\right|_{t=1}$.

Lemma 1. $J(t)$ satisfies the differential equations

$$
\frac{d J}{d t}=\frac{\partial \dot{A^{\ddagger}}}{\partial A} J+\hat{\pi}
$$

and

$$
\frac{d J}{d t}=\frac{\partial \dot{A}^{*}}{\partial A} J+\hat{\pi}^{*}
$$

with initial conditions $J(0)=0$.

We remark that existence and uniqueness are guaranteed due to analyticity of the system.

Proof. We have $J(t)$ as the Jacobian $\partial A(t) / \partial \alpha$. Now, for $A(t)=A(t, \alpha)$, as a function of the variables $t$ and the $\alpha_{i}$, differentiation with respect to $t$ commutes with the partial derivative operators $\partial / \partial \alpha_{i}$. Start with $\dot{A}_{i}=\alpha_{\mu} \tilde{\pi}_{\mu i}(A)$, where we will specialize $\tilde{\pi}$ when needed. Then we have

$$
\frac{d}{d t} \frac{\partial A_{i}(t)}{\partial \alpha_{j}}=\frac{\partial}{\partial \alpha_{j}} \frac{d A_{i}(t)}{d t}=\tilde{\pi}_{j i}(A(t))+\alpha_{\lambda} \frac{\partial \tilde{\pi}_{\lambda i}}{\partial A_{\mu}} \frac{\partial A_{\mu}}{\partial \alpha_{j}} .
$$

Specializing $\tilde{\pi}=\pi^{\ddagger}$, we have, using the definition of $\frac{\partial \dot{A}^{\ddagger}}{\partial A}$,

$$
\dot{J}=\hat{\pi}+\left(\frac{\partial \dot{A}^{\ddagger}}{\partial A}\right)_{i \mu} \frac{\partial A_{\mu}}{\partial \alpha_{j}}
$$

Similarly, $\tilde{\pi}=\pi^{*}$ gives the second formulation.

Next, we observe that the "variation of constants" approach to solving an inhomogeneous linear equation works even in the noncommutative case. That is, suppose we wish to solve $\dot{J}=M J+F$. Writing $J=P Q$, where $P$ is a solution of the homogeneous system, $\dot{P}=M P$, we get $\dot{J}=M J+P \dot{Q}$. In other words, solving the systems $\dot{P}=M P$ and $\dot{Q}=P^{-1} F$ gives us the solution in the form $J=P Q$.

To solve the homogeneous system $\dot{P}=\frac{\partial \dot{A}}{\partial A} P$, we need a preparatory lemma. 
LEMMA 2. The following identities hold:

$$
\xi_{i}^{\ddagger} \pi_{j k}^{*}=\xi_{j}^{*} \pi_{i k}^{\ddagger}
$$

Proof. All of the vector fields $\xi_{i}^{\ddagger}$ commute with every vector field $\xi_{j}^{*}$ since $\xi_{i}^{\ddagger}$ comes from multiplying $g(A, \xi)$ by $\xi_{i}$ on the left and $\xi_{j}^{*}$ by multiplying by $\xi_{j}$ on the right. We get

$$
0=\left[\xi_{i}^{\ddagger}, \xi_{j}^{*}\right]=\left[\pi_{i \lambda}^{\ddagger} \partial_{\lambda}, \pi_{j \mu}^{*} \partial_{\mu}\right]
$$

or

$$
\pi_{i \lambda}^{\ddagger} \frac{\partial \pi_{j \mu}^{*}}{\partial A_{\lambda}} \partial_{\mu}=\pi_{j \mu}^{*} \frac{\partial \pi_{i \lambda}^{\ddagger}}{\partial A_{\mu}} \partial_{\lambda} .
$$

Comparing coefficients of $\partial_{k}$ yields

$$
\pi_{i \lambda}^{\ddagger} \frac{\partial \pi_{j k}^{*}}{\partial A_{\lambda}}=\pi_{j \mu}^{*} \frac{\partial \pi_{i k}^{\ddagger}}{\partial A_{\mu}}
$$

and, recombining terms, the result follows.

Now we can solve the homogeneous systems. Namely,

LEMma 3. Along the path $A(t)$, using the equations $\dot{A}=\alpha \pi^{\ddagger}(A)$, we have

$$
\frac{d \hat{\pi}^{*}}{d t}=\frac{\partial \dot{A}^{\ddagger}}{\partial A} \hat{\pi}^{*}
$$

with initial condition $\hat{\pi}^{*}(0)=I$, the identity matrix. Along the path $A(t)$, using the equations $\dot{A}=\alpha \pi^{*}(A)$, we have

$$
\frac{d \hat{\pi}}{d t}=\frac{\partial \dot{A}^{*}}{\partial A} \hat{\pi}
$$

with initial condition $\hat{\pi}(0)=I$.

First, some notation. Corresponding to basis vector fields $\tilde{\xi}_{i}=\tilde{\pi}(A)_{i \mu} \partial_{\mu}$, we have a typical vector field

$$
\tilde{X}=\alpha_{\lambda} \tilde{\xi}_{\lambda}=\alpha_{\lambda} \tilde{\pi}(A)_{\lambda \mu} \partial_{\mu}
$$

appropriately specialized for $\pi^{\ddagger}$ and $\pi^{*}$.

Proof. According to the characteristic equations $\dot{A}=\alpha \tilde{\pi}(A)$, for any smooth function $f$, $u(t)=f(A(t))$ satisfies

$$
\frac{d u}{d t}=\alpha_{\lambda} \tilde{\pi}_{\lambda \mu}(A(t)) \frac{\partial f}{\partial A_{\mu}}=\tilde{X} u
$$

In particular, $u=\hat{\pi}(A(t))$ satisfies $\frac{d u}{d t}=X^{*} u$. This reads

$$
\frac{d \hat{\pi}_{i k}}{d t}=\alpha_{\lambda} \xi_{\lambda}^{*} \hat{\pi}_{i k}
$$

Using Lemma 2,

$$
\frac{d \hat{\pi}_{i k}}{d t}=\alpha_{\lambda} \xi_{\lambda}^{*} \pi_{k i}^{\ddagger}=\alpha_{\lambda} \xi_{k}^{\ddagger} \pi_{\lambda i}^{*}=\alpha_{\lambda} \pi_{k \mu}^{\ddagger} \partial_{\mu} \pi_{\lambda i}^{*}=\left(\frac{\partial \dot{A}^{*}}{\partial A}\right)_{i \mu} \hat{\pi}_{\mu k} .
$$

The proof for $\hat{\pi}^{*}$ follows exactly the same lines. 
Now consider the equation

$$
\frac{d J}{d t}=\frac{\partial \dot{A}^{*}}{\partial A} J+\hat{\pi}^{*} .
$$

The homogeneous system is solved by $\hat{\pi}$. We are left with solving

$$
\dot{Q}=\hat{\pi}^{-1} \hat{\pi}^{*}
$$

with zero initial conditions. Recalling equation (1), we have $\dot{Q}=\check{\pi}$, which integrates up to $Q(t)=\int_{0}^{t} \check{\pi}(A(s)) d s$. We thus get

$$
J(t)=\hat{\pi}(A(t)) \int_{0}^{t} \check{\pi}(A(s)) d s
$$

and the Theorem follows setting $t=1$. The other pair of equations, starting with $\hat{\pi}^{*}$ for the homogeneous system, gives the second form of the solution.

REMARK. It is natural to wonder what is the effect of a change-of-basis of the Lie algebra on the Jacobian. At this point it is not clear, as in general it appears to involve possibly the entire group law. E.g., if $\xi_{i}=\sum_{j} b_{i j} \eta_{j}$ for some basis $\left\{\eta_{j}\right\}$, then each exponential factor $\exp \left(A_{i} \xi_{i}\right)$ splits according to coordinates of the second kind corresponding to the $\eta$-basis, which would involve the group law expressed in those coordinates. Another way to look at this is to note that the structure constants transform like a quadratic form under change-of-basis. So how do the $A$-coordinates depend on the structure constants? We (with U. Franz) had developed a diagrammatic approach to this problem based on ideas of J. Kocik, but this work has not been completed. In Chapter 2, Theorem 1.1 of $[3$, p. 29], we showed that the structure constants appear in the second-order terms of the expansion of $A$ in terms of $\alpha$. Higher-order terms are nonlinear in the structure constants so it is not clear how they transform. A simple example, that of permuting the basis, is illustrated in [1, pp. 209-212], for the Schrödinger algebra.

4. Examples. Now let us look at details for some Lie algebras of particular interest.

Note. In these examples $\partial$ and $\partial_{i}$ refer to partial derivatives with respect to the $x$ variables.

4.1. Affine Lie algebra. Consider the two-dimensional affine algebra with basis satisfying the commutation relations $\left[\xi_{1}, \xi_{2}\right]=\xi_{2}$. For a matrix realization we take

$$
\xi_{1}=\left(\begin{array}{ll}
1 & 0 \\
0 & 0
\end{array}\right), \quad \xi_{2}=\left(\begin{array}{ll}
0 & 1 \\
0 & 0
\end{array}\right) .
$$

Exponentiating, we have group elements

$$
g(A, \xi)=\left(\begin{array}{cc}
e^{A_{1}} & e^{A_{1}} A_{2} \\
0 & 1
\end{array}\right)
$$

With $X=\alpha_{1} \xi_{1}+\alpha_{2} \xi_{2}$, we have, with $g=\exp (t X)$,

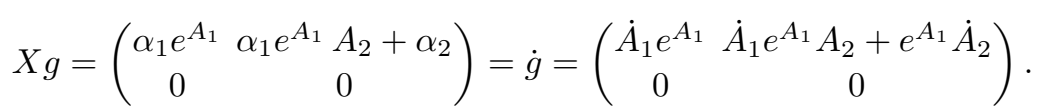


We read off the equations $\dot{A}_{1}=\alpha_{1}, \dot{A}_{2}=\alpha_{2} e^{-A_{1}}$. Comparing with $\dot{A}=\alpha \pi^{\ddagger}(A)$ yields $\pi^{\ddagger}$. Multiplying by $X$ on the right, one similarly finds $\pi^{*}$. The pi-matrices are thus

$$
\pi^{\ddagger}=\left(\begin{array}{cc}
1 & 0 \\
0 & e^{-A_{1}}
\end{array}\right), \quad \pi^{*}=\left(\begin{array}{cc}
1 & -A_{2} \\
0 & 1
\end{array}\right)
$$

and the double dual representation is $\hat{\xi}_{1}=\mathcal{R}_{1}, \hat{\xi}_{2}=\mathcal{R}_{2} e^{-\mathcal{V}_{1}}$. From equation (1), the adjoint group element is

$$
\check{\pi}=\left(\begin{array}{cc}
1 & 0 \\
-A_{2} e^{A_{1}} & e^{A_{1}}
\end{array}\right) .
$$

The coordinate map $\alpha \rightarrow A$ is

$$
A_{1}(\alpha)=\alpha_{1}, \quad A_{2}(\alpha)=\frac{\alpha_{2}}{\alpha_{1}}\left(1-e^{-\alpha_{1}}\right) .
$$

One can use Theorem 1 to find the Jacobian, or one can differentiate directly. We have

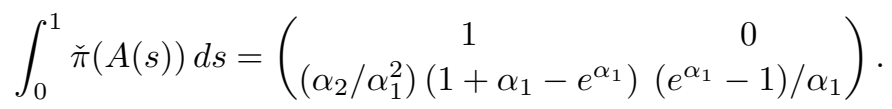

We can write this in terms of the $A$-variables as

$$
\hat{\pi}^{-1} J=\left(\begin{array}{cc}
1 & 0 \\
-\left(A_{2} / A_{1}\right) e^{A_{1}}+A_{2} e^{A_{1}} /\left(e^{A_{1}}-1\right) & \left(e^{A_{1}}-1\right) / A_{1}
\end{array}\right) .
$$

In other words, multiplying by $\hat{\pi}$ :

$$
J=\left(\begin{array}{cc}
1 & 0 \\
-A_{2} / A_{1}+A_{2} /\left(e^{A_{1}}-1\right) & \left(1-e^{-A_{1}}\right) / A_{1}
\end{array}\right) .
$$

Expressing $J$ in terms of $\alpha$-variables, one finds

$$
J^{-1}=\left(\begin{array}{cc}
1 & 0 \\
\alpha_{2} / \alpha_{1}+\alpha_{2} /\left(1-e^{\alpha_{1}}\right) & \alpha_{1} /\left(1-e^{-\alpha_{1}}\right)
\end{array}\right) .
$$

Contracting with $x$ and replacing $A$ by $\partial$ in equation (2) yields the canonical raising operators

$$
\hat{Y}_{1}=x_{1}+x_{2} \partial_{2}\left(\frac{1}{e^{\partial_{1}}-1}-\frac{1}{\partial_{1}}\right), \quad \hat{Y}_{2}=x_{2}\left(\frac{1-e^{-\partial_{1}}}{\partial_{1}}\right) .
$$

These are commuting variables satisfying

$$
\exp \left(\alpha_{1} \hat{Y}_{1}+\alpha_{2} \hat{Y}_{2}\right) 1=\exp \left(x_{1} A_{1}(\alpha)+x_{2} A_{2}(\alpha)\right)
$$

Contracting with $\hat{Y}$ and replacing $\alpha$ by $\mathcal{V}$ in equation (3) yields the $x$-variables in terms of raising and lowering operators

$$
x_{1}=\hat{Y}_{1}+\hat{Y}_{2} \mathcal{V}_{2}\left(\frac{1}{1-e^{\mathcal{V}_{1}}}+\frac{1}{\mathcal{V}_{1}}\right), \quad x_{2}=\hat{Y}_{2}\left(\frac{\mathcal{V}_{1}}{1-e^{-\mathcal{V}_{1}}}\right)
$$

Finally, in equations (4), replace $\hat{Y}$ by $x, \mathcal{V}$ by $\partial$ and contract with the transpose of $\pi^{\ddagger}(A(\partial))$ to yield dual vector fields which satisfy the affine algebra commutation relations yet their exponential when applied to the vacuum is $\exp \left(\alpha_{1} x_{1}+\alpha_{2} x_{2}\right)$ :

$$
\hat{\xi}_{1}=x_{1}+x_{2} \partial_{2}\left(\frac{1}{1-e^{\partial_{1}}}+\frac{1}{\partial_{1}}\right), \quad \hat{\xi}_{2}=x_{2} \frac{\partial_{1}}{e^{\partial_{1}}-1} .
$$


4.2. Finite-difference algebra. The FD algebra is essentially the upper corner of $2 \times 2$ matrices including the diagonal. In other words, we have as a generic element

$$
X=\alpha_{1} \xi_{1}+\alpha_{2} \xi_{2}+\alpha_{3} \xi_{3}=\left(\begin{array}{cc}
\alpha_{3} & \alpha_{2} \\
0 & \alpha_{1}
\end{array}\right) \text {. }
$$

The group element is

$$
g(A, \xi)=\left(\begin{array}{cc}
e^{A_{3}} & A_{2} \\
0 & e^{A_{1}}
\end{array}\right) .
$$

One finds the pi-matrices

$$
\pi^{\ddagger}=\left(\begin{array}{ccc}
1 & 0 & 0 \\
0 & e^{A_{1}} & 0 \\
0 & A_{2} & 1
\end{array}\right), \quad \pi^{*}=\left(\begin{array}{ccc}
1 & A_{2} & 0 \\
0 & e^{A_{3}} & 0 \\
0 & 0 & 1
\end{array}\right) .
$$

Using $\pi^{\ddagger}$, we find the coordinate map

$$
A_{1}=\alpha_{1}, \quad A_{2}=\frac{\alpha_{2}}{\alpha_{1}-\alpha_{3}}\left(e^{\alpha_{1}}-e^{\alpha_{3}}\right), \quad A_{3}=\alpha_{3}
$$

The adjoint group element is

$$
\check{\pi}=\left(\begin{array}{ccc}
1 & 0 & 0 \\
A_{2} e^{-A_{1}} & e^{A_{3}-A_{1}} & -A_{2} e^{-A_{1}} \\
0 & 0 & 1
\end{array}\right) .
$$

The Jacobians take the form

$$
\frac{\partial A}{\partial \alpha}(A)=\left(\begin{array}{ccc}
1 & 0 & 0 \\
\frac{-A_{2}}{A_{1}-A_{3}}+\frac{e^{A_{1}} A_{2}}{e^{A_{1}}-e^{A_{3}}} & \frac{e^{A_{1}}-e^{A_{3}}}{A_{1}-A_{3}} & \frac{A_{2}}{A_{1}-A_{3}}-\frac{e^{A_{3}} A_{2}}{e^{A_{1}}-e^{A_{3}}} \\
0 & 0 & 1
\end{array}\right)
$$

and

$$
\frac{\partial \alpha}{\partial A}(\alpha)=\left(\begin{array}{ccc}
1 & 0 & 0 \\
\frac{\alpha_{2}}{\alpha_{1}-\alpha_{3}}-\frac{e^{\alpha_{1}} \alpha_{2}}{e^{\alpha_{1}}-e^{\alpha_{3}}} & \frac{\alpha_{1}-\alpha_{3}}{e^{\alpha_{1}}-e^{\alpha_{3}}} & \frac{-\alpha_{2}}{\alpha_{1}-\alpha_{3}}+\frac{e^{\alpha_{3}} \alpha_{2}}{e^{\alpha_{1}}-e^{\alpha_{3}}} \\
0 & 0 & 1
\end{array}\right)
$$

From this data one can construct canonical raising operators, express $x$-variables in terms of canonical variables, and construct a representation of the Lie algebra whose exponential acts on the vacuum the same as an abelian algebra.

5. Conclusion. There are many points for continued study. By specializing the coordinates one can find certain elements of the Lie algebra that generate classically interesting polynomials, such as Hermite polynomials via the Heisenberg algebra. In any case, the polynomials found in the approach indicated here have particular structure depending on their associated Lie algebra. Exactly how the polynomials and the structure of the Lie algebra are related in some deeper way has not been clarified.

Another source of interest is, of course, the Jacobians. One can look at Jacobians of the form $\partial A(t) / \partial A(s)$, for $s<t$. As the Jacobians form a multiplicative family along paths, 
there are some possibilities for interesting dynamical systems, or perhaps, matrix-valued stochastic processes.

Generally speaking, it looks challenging and interesting to get some detailed information for classes of higher-dimensional Lie algebras. Certain classes of Lie algebras, such as symmetric Lie algebras, may allow for general structural results.

\section{References}

[1] Ph. Feinsilver and R. Schott, Dual representations for the Schrödinger algebra, in: Quantum Probability and Infinite-Dimensional Analysis, World Scientific, 2005, 201-215.

[2] Ph. Feinsilver and R. Schott, Vector fields and their duals, Adv. in Math. 149 (2000), $182-192$.

[3] Ph. Feinsilver and R. Schott, Algebraic Structures and Operator Calculus, Volume 3: Representations of Lie Groups, Kluwer Academic Publishers, 1996. 